\title{
The influence of pore fluids on seismic wave velocities under high temperature and high pressure conditions: Development of a new technique with gas apparatus at AIST, Japan
}

\author{
Keigo Kitamura $^{1 *}$, Koji Masuda ${ }^{1}$, Miki Takahashi $^{1}$, and Osamu Nishizawa ${ }^{2}$ \\ ${ }^{1}$ Institute of Geology and Geoinformation, Geological Survey of Japan, National Institute of Advanced Industrial Science and \\ Technology (AIST), AIST Tsukuba Central 7, Tsukuba, 305-8567, Japan \\ ${ }^{2}$ Institute for Geo-Resources and Environment, Geological Survey of Japan, National Institute of Advanced Industrial \\ Science and Technology (AIST), AIST Tsukuba Central 7, Tsukuba, 305-8567, Japan
}

(Received March 13, 2006; Revised July 4, 2006; Accepted July 14, 2006; Online published December 25, 2006)

\begin{abstract}
In order to study the effects of different pore fluids on elastic-wave velocities, we developed a new system for measuring elastic wave velocities $\left(V_{p}\right.$ and $\left.V_{s}\right)$ under high-pressure conditions up to $200 \mathrm{MPa}$ and hightemperature conditions up to $200^{\circ} \mathrm{C}$ using a gas-medium high-pressure and high-temperature apparatus. During the $V_{p}$ and $V_{s}$ measurements, we can also control the pore pressures from outside the rock samples. We measured $V_{p}$ and $V_{s}$ of the Berea sandstone samples under three different pore-fluid conditions including dry (without porefluids), in the presence of pore water, and in the presence of pore gas. Here we describe our new measurement technique and establish the capacity of the gas apparatus and measurement technique and their potentialities for the solution of geological problems.
\end{abstract}

Key words: Pore fluid, $V_{p}, V_{s}$, Berea sandstone, gas apparatus.

\section{Introduction}

Fluids with various kinds of chemical compositions exist everywhere on Earth. It is well known that the physical properties of rocks that are components of the Earth's crust are strongly affected by the presence of crustal fluids. For example, the existence of water is believed to be responsible for the low velocities based on laboratory measurements of the seismic velocities of water-saturated rocks under high confining pressure and temperature conditions. The chemical variations of pore fluids also strongly affect elastic-wave velocities (Wulff and Burkhardt, 1997). The effects of temperature on the elastic-wave velocities of saturated rocks have also been observed (e.g., Matsushima, 1981; Kern and Richiter, 1981; Ito and Tatsumi, 1995; Zharikov et al., 2000).

Laboratory measurements conducted by Christensen (1984) and Christensen and Wang (1985) showed that pore pressure also contributes significantly to the low velocity and lateral variability in seismic velocity structures. In order to understand the effects of crustal fluids on the physical properties of rocks such as seismic-wave velocities, we need to measure the effects of fluids on the elastic-wave velocities under controlled pore pressure conditions, because the pore pressure strongly affects the elastic-wave velocities. Laboratory data on the seismic-wave velocities

*Now at the Research Institute of Innovative Technology for the Earth (RITE), 9-2 Kizugawadai, Kizu-cho, Soraku-gun, Kyoto 619-092, Japan.

Copyright (c) The Society of Geomagnetism and Earth, Planetary and Space Sciences (SGEPSS); The Seismological Society of Japan; The Volcanological Society of Japan; The Geodetic Society of Japan; The Japanese Society for Planetary Sciences; TERRAPUB of rocks measured under in-situ conditions such as high pressure, high temperature, and well controlled pore-fluid pressure are essential when we interpret the recent highprecision seismic-tomography results. Although their importance is well recognized, there have been few reports on the elastic-wave velocities of rocks measured under controlled pore-fluid pressure.

In the present study, we designed and developed a new measurement system using a gas-medium, high-pressure and high-temperature deformation apparatus at AIST, Japan (Masuda et al., 2002). We report a new velocity measurement technique under high-pressure and high-temperature conditions in which we can independently control the pore fluids and pore pressures. We show the potential of our methods by presenting the preliminary results on the effects of pore fluids such as water and argon (Ar) gas on the compressional and shear wave velocities $\left(V_{p}\right.$ and $\left.V_{s}\right)$ of Berea sandstone samples under high-temperature and highpressure conditions.

\section{Experimental Procedures}

We have developed a new method for velocity measurement under the in-situ conditions of the crust, which are characterized by high pressure (up to $200 \mathrm{MPa}$ ), high temperature (up to $200^{\circ} \mathrm{C}$ ), and the existence of pore fluids. During the $V_{p}$ and $V_{s}$ measurements, we can also control the pore pressures independently from outside the rock samples. We modified the gas-medium, high-pressure and hightemperature deformation apparatus at AIST, Japan (Fig. 1). With this apparatus, Ar gas is used as a pressure medium for confining pressure. A pore pressure of up to $200 \mathrm{MPa}$ is generated by Ar gas or water. The specimen is continuously 


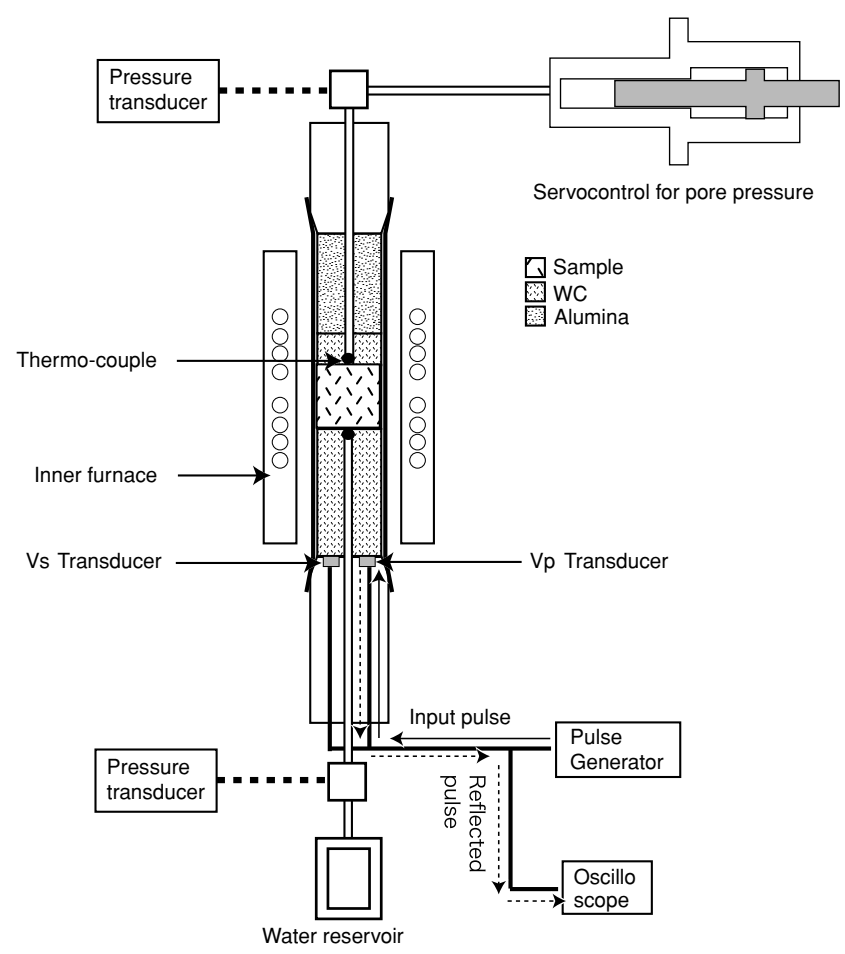

Fig. 1. Schematic illustration of the gas-medium, high-temperature and high-pressure deformation apparatus and sample assembly.

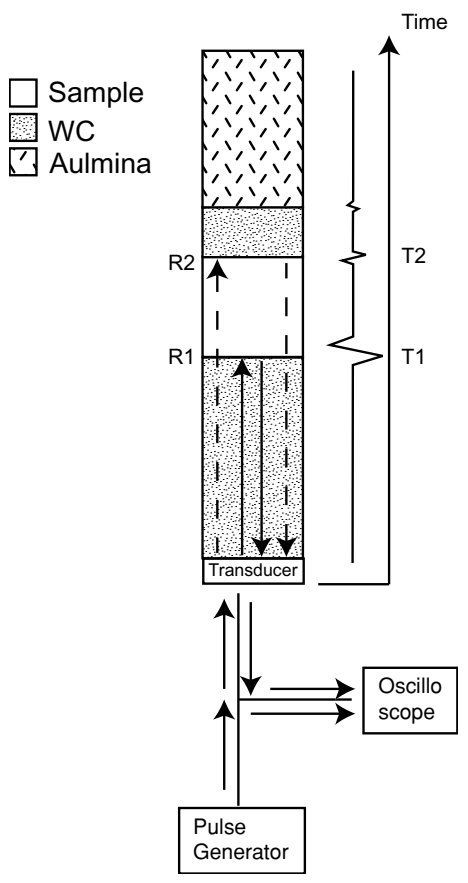

Fig. 2. Schematic diagram showing the ray paths for the pulse reflection method for compressional and shear wave velocity measurement. R1: Reflector-1, R2: Reflector-2.

connected to the pore fluids reservoir ("drained test"). The pore pressure can be applied from outside and controlled independently by a servo-controlled system under various confining-pressure and temperature conditions. The confining pressure was also controlled by the servo-control system (Fig. 1(a)). The precision levels of both pressure controls are within $0.05 \%$.

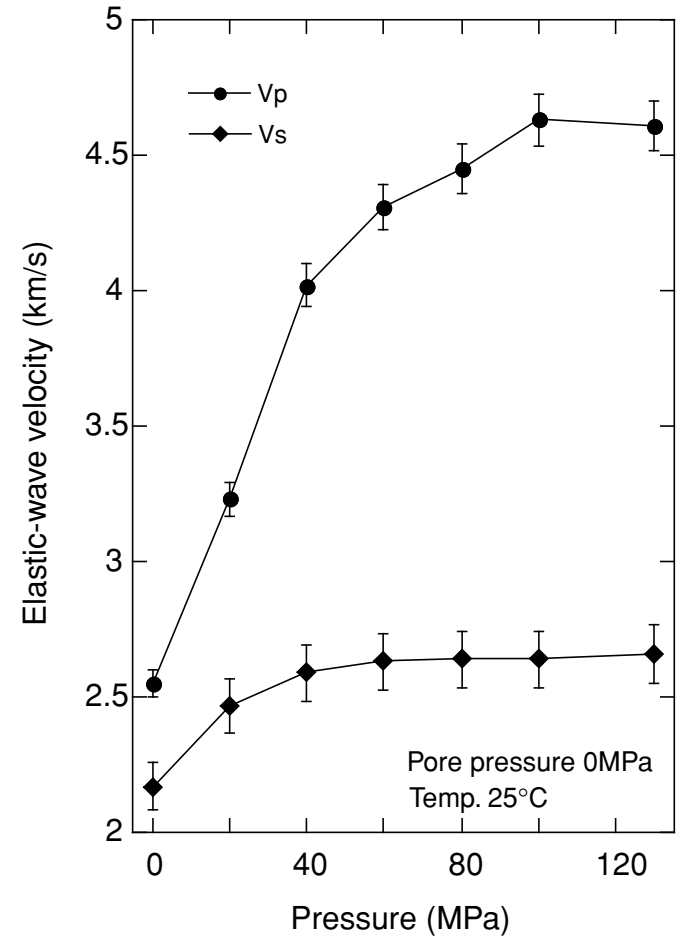

Fig. 3. Elastic-wave velocities $\left(V_{p}\right.$ and $\left.V_{s}\right)$ of the Berea sandstone samples as a function of confining pressure under the dry condition at a temperature of $25^{\circ} \mathrm{C}$.

This apparatus can also control temperature up to $800^{\circ} \mathrm{C}$. We were able to produce the in-situ conditions of the seismogenic zone and carry out various types of experimental studies with this apparatus (e.g., a deformation experiment; Masuda et al., 2002).

We measured the elastic wave velocities for Berea sandstone, which is coarse-grained (average grain size, $70 \mu \mathrm{m}$ ) and has $15-18 \%$ porosity. Because Berea sandstone is porous and previous data are available, we decided to choose this rock for our preliminary tests. This porous rock is suitable for evaluating the effects of pore fluids on elasticwave velocities. The rock samples were cut to a cylindrical shape (20.0 $\mathrm{mm}$ in diameter and $20.0 \mathrm{~mm}$ in length). The end surfaces were made parallel to within $2 / 100 \mathrm{~mm}$.

We measured the $V_{p}$ and $V_{s}$ of the Berea sandstone samples under three different pore-fluid conditions, including without pore-fluids, in the presence of pore water, and in the presence of pore gas. We measured $V_{p}$ and $V_{s}$ without pore fluids in order to evaluate the effects of pore fluids on the elastic-wave velocities. We chose water or Ar gas as saturating fluids in the case of existing pore fluids in order to evaluate the effects of water and inertia-gas as possible end members of pore fluids.

We adopted the pulse reflection method for velocity measurements (e.g., Kono et al., 2004) because our apparatus has a restricted working space in the high pressure vessel. We designed a new lower piston for the pulse reflection method. The lithium niobate $\left(\mathrm{LiNbO}_{3}\right)$ transducers were cut in $36^{\circ} \mathrm{Y}$ for $V_{p}$ and $163^{\circ} \mathrm{Y}$ for $V_{s}$ and used for velocity measurements. Three transducers (with a resonant frequency of $3.3 \mathrm{MHz}$ ) were set at the top of the lower piston for one $P$-wave and two $S$-waves. The sample assembly 

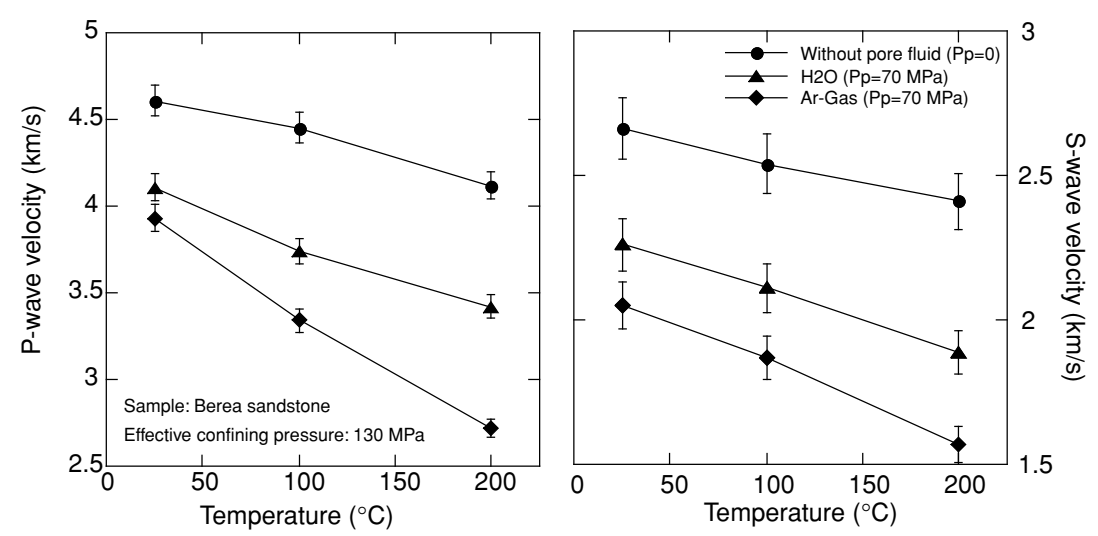

Fig. 4. Elastic-wave velocities $\left(V_{p}\right.$ and $V_{s}$ ) of the Berea sandstone samples as a function of temperature for three pore fluids conditions, dry (without pore fluids), with water, and with Ar-gas. The effective confining pressure of $130 \mathrm{MPa}$ was kept constant for all the measurements.

was placed in a $0.2 \mathrm{~mm}$ thick cupper jacket tube between the tungsten carbide (WC) buffer rod and the reflector plug. This experimental setup was set at the lower piston (Fig. 1). Figure 2 shows the ray path of the experimental design for the pulse reflection method. The elastic waves are generated by the transducers. These elastic wave pulses were partly reflected at Reflector-1, which is the interface between the buffer rod and the sample. The oscilloscope recorded the first arrival that was reflected at Reflector-1 as T1. However, some parts of the pulses passed through the rock sample and were reflected at Reflector-2, which is the interface between the sample and the other reflector plug; the waves reflected at Reflector-2 were recorded as T2. We defined half of the time difference between $\mathrm{T} 2$ and $\mathrm{T} 1$ as the travel time and calculated the elastic wave velocity based on this travel time and the sample length. Received waves were stacked up to 1000 times, and were recorded with an oscilloscope with a sampling rate of $5.0 \times 10^{6}$ samples/s. To determine the travel time precisely, we applied a band pass filter to the stacked-wave data and carried out automatic peak searching in phase. We calibrated the precision of the velocity measurements by measuring the elastic wave velocity of a standard quartz sample with the same shape as the Berea sandstone samples. Errors in our velocity measurements have a maximum of $\pm 2 \%$ in $V_{p}$ and $\pm 4 \%$ in $V_{s}$.

\section{Results}

\section{1 $V_{p}$ and $V_{s}$ for Berea sandstone as a function of pressure}

Figure 3 shows the $V_{p}$ and $V_{s}$ as a function of confining pressure. $V_{p}$ and $V_{s}$ were measured at $20 \mathrm{MPa}$ intervals during pressurization of the confining pressure, $P c$, up to $130 \mathrm{MPa}$ under dry conditions (temperature, $25^{\circ} \mathrm{C}$; pore pressure, $P p, 0 \mathrm{MPa})$. $V_{p}$ and $V_{s}$ increased rapidly with $P c$ to $100 \mathrm{MPa}$ in $V_{p}$ (from $2.55 \mathrm{~km} / \mathrm{s}$ to $4.63 \mathrm{~km} / \mathrm{s}$ ) and 60 $\mathrm{MPa}$ in $V_{s}$ (from $2.17 \mathrm{~km} / \mathrm{s}$ to $2.63 \mathrm{~km} / \mathrm{s}$ ). They increased slightly in higher $P c$ ranges, due to the closures of pores and cracks during pressurization (e.g., Christensen, 1974; Wulff and Burkhardt, 1997; Niesler and Jackson, 1989).

\subsection{Effects of temperature and pore fluids on the elas-} tic wave velocities of Berea sandstone

We measured the $V_{p}$ and $V_{s}$ of Berea sandstone samples under a fixed effective confining pressure of 130
$\mathrm{MPa}$ and with changing temperatures ranging from 25 to $200^{\circ} \mathrm{C}$. The effective confining pressure, $P_{\text {eff }}$, is defined as $P_{\text {eff }}=P c-\alpha P p$, where $P c(130$ or $200 \mathrm{MPa})$ is the confining pressure, $P p(0$ or $70 \mathrm{MPa})$ is the pore fluid (water or Ar gas) pressure, and $\alpha$ is a coefficient (in this study, $\alpha=1)$. Previous studies have indicated that elastic wave velocities are strongly affected by the effective confining pressure (e.g., Todd and Simmons, 1972). Thus, in the present study, $V_{p}$ and $V_{s}$ were measured under the fixed effective confining pressure $\left(P_{\text {eff }}=130 \mathrm{MPa}\right)$ and three temperature conditions (from 25,100 and $200^{\circ} \mathrm{C}$ ) in order to evaluate the effects of pore fluids and the temperature dependence of their elastic properties on $V_{p}$ and $V_{s}$. In order to evaluate the effects of pore fluids on $V_{p}$ and $V_{s}$, measurements were carried out under three conditions, one without pore fluid and two conditions in which cracks were filled, one with Ar gas and one with water. Figure 4 shows the elastic wave velocities $\left(V_{p}\right.$ and $\left.V_{s}\right)$ as a function of temperature for the three pore-fluid conditions. Under room temperature conditions $\left(25^{\circ} \mathrm{C}\right), V_{p}$ and $V_{s}$ showed significant differences among the three conditions (without pore fluid, $V_{p}=4.60 \mathrm{~km} / \mathrm{s}, V_{s}=2.66 \mathrm{~km} / \mathrm{s} ; \mathrm{Ar}$ gas-filled, $V_{p}=3.81 \mathrm{~km} / \mathrm{s}, V_{s}=2.05 \mathrm{~km} / \mathrm{s}$; and water-filled, $\left.V_{p}=4.11 \mathrm{~km} / \mathrm{s}, V_{s}=2.26 \mathrm{~km} / \mathrm{s}\right)$. The elastic wave velocities decreased lineally with increasing temperature (from 25 to $200^{\circ} \mathrm{C}$ ). $V_{p}$ and $V_{s}$ showed larger differences in each run at $200^{\circ} \mathrm{C}$ than at $25^{\circ} \mathrm{C}$ (without pore fluid, $V_{p}=4.12$ $\mathrm{km} / \mathrm{s}, V_{s}=2.41 \mathrm{~km} / \mathrm{s}$; Ar gas-filled, $V_{p}=2.72 \mathrm{~km} / \mathrm{s}, V_{s}=1.57$ $\mathrm{km} / \mathrm{s}$; and water-filled, $V_{p}=3.42 \mathrm{~km} / \mathrm{s}, V_{s}=1.89 \mathrm{~km} / \mathrm{s}$ ). The thermal reduction ratio of elastic wave velocities is strongly controlled by pore fluids (without pore fluid, $V_{p}=10.5 \%$, $V_{s}=9.6 \%$; Ar gas-filled, $V_{p}=28.6 \%, V_{s}=23.4 \%$; and waterfilled, $V_{p}=16.5 \%, V_{s}=16.3 \%$ ).

\section{Discussion and Conclusion}

The different velocities were obtained at the same effective confining pressure under the dry condition (without fluids) and with water or Ar saturation as shown in Fig. 4. In this study, the values of effective pressure during all of the experiments were kept to the same as $130 \mathrm{MPa}$. Because we could not observe the alternation of the grain shape of composite minerals in the thin sections of the samples after the experiments, the effects of chemical reactions such as ther- 
mal decomposition, pressure-solution, the forming of new minerals, phase transfers and other processes are thought to be negligible in those measurements. These data show that the Ar gas velocities are lower than the water velocities. It is possible that the observed differences in velocities can be explained by calculating the effective elastic moduli of the saturated rock as the moduli of a composite medium made of rock and inclusions. The temperature effect on the velocities is also shown clearly in the range from room temperature to $200^{\circ} \mathrm{C}$, and it entails the decrease of the elastic constants of the minerals and fluid inclusion as the temperature increases.

This new experimental method of measuring the elasticwave velocities of rocks under controlled pore fluid pressure and temperature conditions was developed in order to study the effects of different pore fluids on $V_{p}$ and $V_{s}$. We concluded that our new measurement system has the potential to be very useful in helping to solve geological problems.

Acknowledgments. We used the software coded by X. Lei of the GSJ, AIST to record and stack the elastic waves. The technical work of K. Iryo and A. Ogura of PRETECH was very helpful in developing and implementing the pulse reflection technique inside the gas-medium pressure vessel. Reviews of Drs. A. V. Zharikov, and Y. Gueguen were very helpful in improving the manuscript.

\section{References}

Christensen, N. I., Compressional wave velocities in possible mantle rock to pressure of 30 kilobars, J. Geophys. Res., 79, 407-412, 1974.

Christensen, N. I., Pore pressure and oceanic crustal seismic structure,
Geophys. J. R. Astr. Soc., 79, 411-423, 1984.

Christensen, N. I. and H. F. Wang, The influence of pore pressure and confining pressure on dynamic elastic properties of Berea sandstone, Geophysics, 50, 207-213, 1985.

Ito, K. and Y. Tatsumi, Measurement of elastic wave velocities in granulite and amphibolite having identical $\mathrm{H}_{2} \mathrm{O}$-free bulk compositions up to $850^{\circ} \mathrm{C}$ at $1 \mathrm{GPa}$, Earth. Planet. Sci. Lett., 133, 255-264, 1995.

Kern, H. and A. Richter, Temperature derivatives of compressional and shear wave velocities in crustal and mantle rocks at 6 kbar confining pressure, J. Geophys., 49, 47-56, 1981.

Kono, Y., M. Ishikawa, and M. Arima, Discontinuous change in temperature derivative of $V_{p}$ in lower crustal rocks, Geophys. Res. Lett., 31, L22601, 2004.

Masuda, K., K. Fujimoto, and T. Arai, A new gas-medium, high-pressure and high-temperature deformation apparatus at AIST, Japan, Earth Planets Space, 54, 1091-1094, 2002.

Matsushima, S., Compressional and shear wave velocities of igneous rocks and volcanic glasses to $900^{\circ} \mathrm{C}$ and $20 \mathrm{Kbar}$, Tectonophysics, 75, 257$271,1981$.

Niesler, H. and I. Jackson, Pressure derivatives of elastic wave velocities from ultrasonic interferometric measurements on jacketed polycrystals, J. Acoust. Soc. Am., 86, 1573-1585, 1989.

Todd, T. and G. Simmons, Effect of pore pressure on the velocity of compressional wave in low porosity rocks, J. Geophys. Res., 77, 37313743, 1972.

Wulff, A.-M. and H. Burkhardt, Mechanisms affecting ultrasonic wave propagation in fluid-containing sandstone under high hydrostatic pressure, J. Geophys. Res., 102, 3043-3050, 1997.

Zharikov, A. V., E. B. Lebedev, A. M. Dorfman, and V. M. Vitovtova, Effect of saturating fluid composition on the rock microstructure, permeability and $V_{p}$ under high pressure and temperature, Phys. Chem. Earth (A), 25, 215-218, 2000.

K. Kitamura (e-mail: keigo@rite.or.jp), K. Masuda, M. Takahashi, and O. Nishizawa 